\title{
Wavelet based Multi Class image classification using Neural Network
}

\author{
Ajay Kumar Singh \\ MITS Deemed University \\ Lakshmangarh, Sikar \\ Rajasthan, India
}

\author{
Shamik Tiwari \\ MITS Deemed University \\ Lakshmangarh, Sikar \\ Rajasthan, India
}

\author{
V.P. Shukla \\ MITS Deemed University \\ Lakshmangarh, Sikar \\ Rajasthan, India
}

\begin{abstract}
This paper presents feature extraction and classification of multiclass images by using Haar wavelet transform and back propagation neural network. The wavelet features are extracted from original texture images and corresponding complementary images. The features are made up of different combinations of sub-band images, which offer better discriminating strategy for image classification and enhance the classification rate.
\end{abstract}

\section{General Terms}

Computer Vision, Pattern Recognition

\section{Keywords}

Multi class, Wavelet decomposition, Feature Extraction, Neural Network, Haar wavelet

\section{INTRODUCTION}

Multi class image classification plays an important role in many computer vision applications such as biomedical image processing, automated visual inspection, content based image retrieval, and remote sensing applications. Image classification algorithms can be designed by finding essential features which have strong discriminating power, and training the classifier to classify the image. Scientists and practitioners have made great efforts in developing advanced classification approaches and techniques for improving classification accuracy $[1,2,3,4,5$ and 6$]$.

Wavelets are mathematical functions which help in describing the original image into an image in frequency domain, which can further divided into subband images of different frequency components. Each component is studied with a resolution matched to its scale. Wavelet transform has advantages over traditional Fourier method in analyzing physical situations where the signal contains discontinuities and sharp spikes.

In the past two decades, the wavelet transforms are being applied successfully in various applications, such as feature extraction and classification. Wavelet transforms and their variants such as wavelet packets have become an appropriate starting point for classification, especially for texture images $[7,8]$, due to scale-space localization properties of. Also the choice of filters in the wavelet transform plays an important role in extracting the features of texture images $[7,8]$.

Neural networks are used as statistical tools in various fields, including Statistics, Engineering, Psychology, Physics and also Economics. The goal of the neural network is to learn or to discover some association between input and output patterns, or to analyze, or to find the structure of the input patterns. The learning process is achieved through the modification of the connection weights between units. In the work [9] describes multi spectral classification of land-sat images using neural networks. The work in [10] describes the classification of multi spectral remote sensing data using a Back-propagation Neural Network. A comparison to conventional supervised classification by using minimal training set in Artificial Neural Network is given in [11]. Remotely sensed data by using Artificial Neural Network based have been classified in [12] on software package. In [13] different types of noise are classified using feed forward neural network.

In this paper a wavelet feature extraction algorithm is proposed for image classification problem. Here cumulative histograms are generated using different combinations of approximations and detail sub bands of the wavelet decomposed image and then different features are extracted.

This paper is organized as follows: In the next section Wavelet decomposition using Haar wavelet is discussed. Feed Forward Neural Network is discussed in section 3. In section 4 feature extraction algorithm is discussed. Training and classification is discussed in 5 and finally result is discussed in section 6 .

\section{HAAR WAVELET RANSFORMATION}

The wavelet transform was developed for further developments from Fourier transforms. Wavelets transform signals in the time domain (rather, assumed to be in the time domain) to a joint time-frequency domain. The main weakness that was found in Fourier transforms was their lack of localized support. In short, this means that we could get information about the frequencies present in a signal, but not where and when the frequencies occurred. The first DWT was invented by the Hungarian mathematician Alfréd Haar. For an input represented by a list of $2^{\mathrm{n}}$ numbers, the Haar wavelet transform may be considered to simply pair up input values, storing the difference and passing the sum. This process is repeated recursively, pairing up the sums to provide the next scale: finally resulting in $2^{\mathrm{n}}-1$ difference and one final sum. The Haar DWT illustrates the desirable properties of wavelets in general. First, it can be performed in $\mathrm{O}(\mathrm{n})$ operations; second, it captures not only a notion of the frequency content of the input, by examining it at different scales, but also temporal content, i.e. the times at which these frequencies occur. Combined, these two properties make the Fast wavelet transform (FWT) an alternative to the conventional Fast Fourier Transform (FFT). FWT is used for signal coding, to represent a discrete signal in a more redundant form, often as a preconditioning for data compression [14]. 
The Haar transformation technique is used [15] to form a wavelet since it is the simplest wavelet transformation method of all and can effectively serve our interests. In the Haar wavelet transformation method, low-pass filtering is conducted by averaging two adjacent pixel values, whereas the difference between two adjacent pixel values is figured out for high-pass filtering. The Haar wavelet applies a pair of low-pass and high-pass filters to image decomposition first in image columns and then in image rows independently. As a result, it produces four sub-bands as the output of the first level Haar wavelet. The four sub-bands are LL1, HL1, LH1, and HH1. The low-frequency sub-band LL1 can be further decomposed into four sub-bands LL2, HL2, LH2, and HH2 at the next coarser scale. LLi is a reduced resolution corresponding to the low frequency part of the image. The other three sub-bands $\mathrm{HLi}, \mathrm{LHi}$ and Hhi are the high frequency parts in the vertical, horizontal, and diagonal directions, respectively [8].

The Haar transform cross-multiplies a function against the Haar wavelet with various shifts and stretches, like the Fourier transform cross-multiplies a function against a sine wave with two phases and many stretches. The Haar transform is derived from the Haar matrix. An example of a $4 \times 4$ Haar matrix is shown below [16]:

$$
H_{4}=\frac{1}{\sqrt{ } 4}\left[\begin{array}{cccc}
1 & 1 & 1 & 1 \\
1 & 1 & -1 & -1 \\
\sqrt{2} & -\sqrt{2} & 0 & 0 \\
0 & 0 & \sqrt{2} & -\sqrt{2}
\end{array}\right]
$$

\section{FEEDFORWARD NEURAL}

\section{NETWORK}

A successful pattern classification methodology [17] depends heavily on the particular choice of the features used by the classifier . The Back-Propagation is the best known and widely used learning algorithm in training multilayer feed forward neural networks. The feed forward neural net refer to the network consisting of a set of sensory units (source nodes) that constitute the input layer, one or more hidden layers of computation nodes, and an output layer of computation nodes. The input signal propagates through the network in a forward direction, from left to right and on a layer-by-layer basis. Back propagation is a multi-layer feed forward, supervised learning network based on gradient descent learning rule. This BPNN provides a computationally efficient method for changing

the weights in feed forward network, with differentiable activation function units, to learn a training set of input-output data. Being a gradient descent method it minimizes the total squared error of the output computed by the net. The aim is to train the network to achieve a balance between the ability to respond correctly to the input patterns that are used for training and the ability to provide good response to the input that are similar. A typical back propagation network of input layer, one hidden layer and output layer is shown in figure 1 .

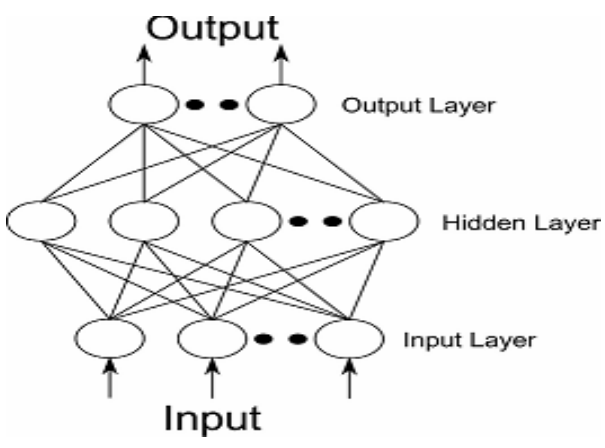

Fig 1: Feed Forward BPN

The steps in the BPN training algorithm are:

Step 1: Initialize the weights.

Step 2: While stopping condition is false, execute step 3 to 10.

Step 3: For each training pair x:t, do steps 4 to 9.

Step 4: Each input unit $X i, i=1,2, \ldots, n$ receives the input signal, $\mathrm{x}_{\mathrm{i}}$ and broadcasts it to the next layer.

Step 5: For each hidden layer neuron denoted as $Z_{j}, j=$ $1,2, \ldots, p$.

$$
\begin{aligned}
& z_{i n j}=v_{o j}+\sum_{i} x_{i} v_{i j} \\
& z_{j}=f\left(z_{i n j}\right)
\end{aligned}
$$

Broadcast $z_{j}$ to the next layer. Where $\mathrm{v}_{\mathrm{oj}}$ is the bias on $\mathrm{j}^{\text {th }}$ hidden unit.

Step 6: For each output neuron $Y_{k}, k=1,2, \ldots . m$

$$
\begin{aligned}
& \mathrm{y}_{\text {ink }}=w_{o k}+\sum_{j} z_{j} w_{j k} \\
& y_{k}=f\left(y_{\text {ink }}\right)
\end{aligned}
$$

Step 7: Compute $\delta_{k}$ for each output neuron, $Y_{k}$

$$
\begin{aligned}
& \delta_{k}=\left(t_{k}-y_{k}\right) f^{\prime}\left(y_{i n k}\right) \\
& \Delta w_{j k}=\alpha \delta_{k} z_{j} \\
& \Delta w_{o k}=\alpha \delta_{k} \quad \text { since } z_{0}=1
\end{aligned}
$$

Where $\delta_{k}$ is the portion of error correction weight adjustment for $w_{j k}$ i.e. due to an error at the output unit $y_{k}$, which is back propagated to the hidden unit that feed it into the unit $\mathrm{y}_{\mathrm{k}}$ and $\alpha$ is learning rate.

Step 8: For each hidden neuron

$$
\begin{aligned}
& \delta_{i n j}=\sum_{k=1}^{m} \delta_{k} w_{j k} \quad j=1,2, \ldots . . p \\
& \delta_{j}=\delta_{i n j} f^{\prime}\left(z_{i n j}\right) \\
& \Delta v_{i j}=\alpha \delta_{j} x_{i} \\
& \Delta v_{o j}=\alpha \delta_{j}
\end{aligned}
$$


Where $\delta_{j}$ is the portion of error correction weight adjustment for $v_{i j}$ i.e. due to the back propagation of error to the hidden unit $\mathrm{z}_{\mathrm{j}}$

Step 9: Update weights.

$$
\begin{aligned}
& w_{j k}(\text { new })=w_{j k}(\text { old })+\Delta w_{j k} \\
& v_{i j}(\text { new })=v_{i j}(\text { old })+\Delta v_{i j}
\end{aligned}
$$

Step 10: Test for stopping condition.

\section{METHODOLOGY}

Features are the characteristics of the object of interest. Feature extraction methodologies analyze objects and images to extract the most prominent features which are representatives of the various classes of images. Following methodology is used to extract the features of the texture images.

Step 1: Read the RGB image $f(x, y, z)$

Step 2: Convert the image to gray scale image $f(x, y)$.

Step 3: Compute the Haar wavelet coefficients of step2 image, which returns four matrices A, H, V, D corresponding to approximations, horizontal, vertical \& diagonal coefficients of image.

Step 4: For each pair of coefficients $(A, H),(A, V),(A, D)$ and (A-abs(V-H-D)) repeat the step5 to step8.

Suppose first matrix in pair is $\mathrm{X} \&$ second is $\mathrm{Y}$ and get size of matrix is $[\mathrm{M}, \mathrm{N}]$.

Step 5: For I=1 to 8 repeat the step 6. Where I represents the 8 neighbors in each direction of any point as shown in figure 2.

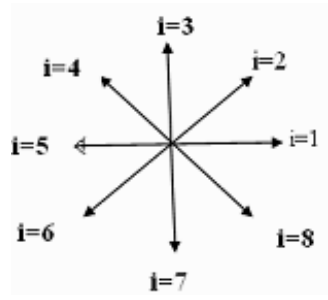

Fig 2: 8 neighbors of any point

Step 6: Repeat for $J=1$ to $M$

Repeat for $\mathrm{K}=1$ to $\mathrm{N}$

$\mathrm{d}=\max (\min (\mathrm{X}(\mathrm{J}, \mathrm{K}), \mathrm{Y}(\mathrm{I})), \min (\mathrm{Y}(\mathrm{J}, \mathrm{K}), \mathrm{X}(\mathrm{I})))$

if $\mathrm{d}=\min (\mathrm{X}(\mathrm{J}, \mathrm{K}))$

Set $\mathrm{H} 1(\mathrm{I})=\mathrm{X}(\mathrm{J}, \mathrm{K})$

Else

Set $\mathrm{H} 2(\mathrm{I})=\mathrm{Y}(\mathrm{J}, \mathrm{K})$

End

End

Step 7: For $\mathrm{I}=1$ to 8 cumulative histogram of $\mathrm{H} 1$ \& $\mathrm{H} 2$ is found shown in the figure 3 .

Step 8: For each cumulative histogram correlation coefficient, mean and standard deviation features are computed.
Step 9: Repeat step3 to step8 for complement image of $f(x, y)$ also.

Step 10: Arrange the features in a matrix.

\section{TRAINING AND CLASSIFICATION}

In this phase of experiment we have divided each texture image in to sixteen blocks of equal size, out of which six blocks are used as samples to train the neural classifier and remaining ten blocks are used as samples to test the texture class. Texture features are extracted using the algorithm discussed in part 4 for each of the six samples.

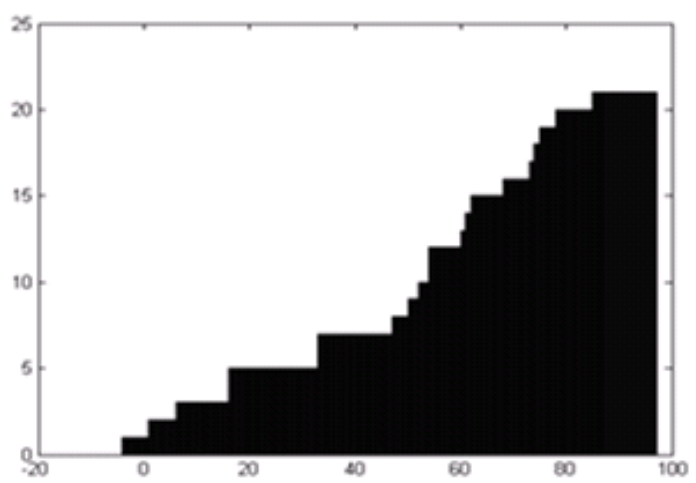

Fig 3: Cumulative histogram

Then neural classifier is trained with this feature set. After training the classifier random samples for test are selected for classification. Features of these samples are also extracted using the same feature extraction algorithm discussed in part 4 , and applied to neural classifier for testing.

The classification is performed over a 5-10-15 feed forward neural network model that consist of one input layer with five neurons, one hidden layer with ten neurons and one output layer with fifteen neurons. Back propagation is pertained as network training principle where the training dataset is constructed by the extracted features of the image. The entire input features are normalized into the range of $[0,1]$, whereas the output class is assigned to one for the highest probability and zero for the lowest.

\section{EXPERIMENTAL RESULT}

\subsection{Data Set}

To assess the capability of wavelet feature based classification of images we have taken the texture images from the database available by the University of Southern California's Signal and Image Processing Institute Volume 1[19]. The test is carried out on 15 categories shown collectively, are selected from the images of Brodatz[19]. Here textured samples are obtained from the image of 64X64 and each image is subdivided into equal sized 16 blocks of size 16 X16 with 256 gray levels. This produced 480 blocks (240 of original and 240 of negative image). Here 180 blocks (90 blocks of original image and 90 blocks of complement) are used to train the neural classifier and from the remaining randomly chosen for testing. 


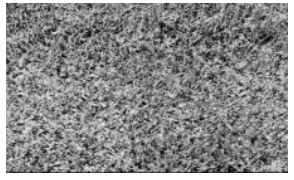

1
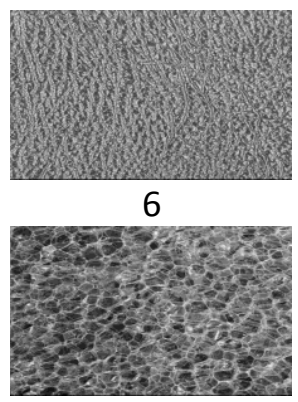

11

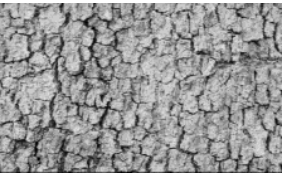

2

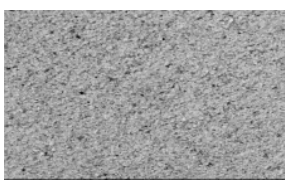

7

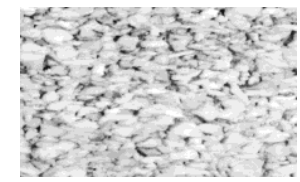

12

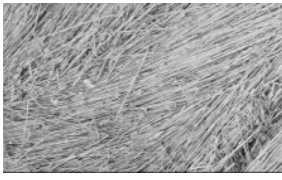

3

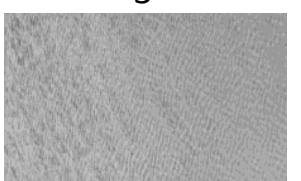

8

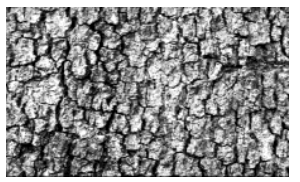

13

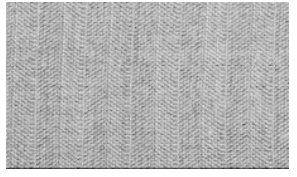

4

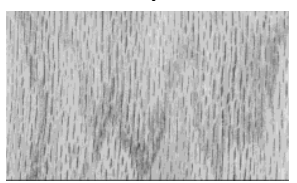

9

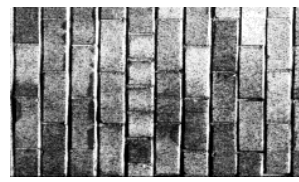

14

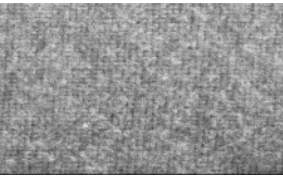

5

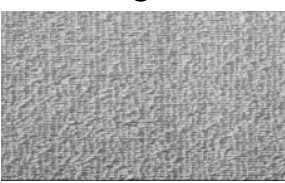

10

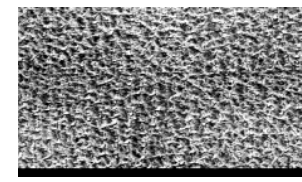

15

Fig 4: Texture Image from Brodatz album

\begin{tabular}{|c|c|c|c|}
\hline S.No. & Texture & $\begin{array}{c}\text { Montiel } \\
\text { et al. } \\
(2005)\end{array}$ & $\begin{array}{c}\text { Proposed } \\
\text { System }\end{array}$ \\
\hline 1 & Grass (D9) & 99.06 & 98.56 \\
\hline 2 & Bark (D12) & 100 & 100 \\
\hline 3 & Straw (D15) & 100 & 100 \\
\hline 4 & $\begin{array}{c}\text { Herringbone weave } \\
\text { (D15) }\end{array}$ & Not Tested & 86.02 \\
\hline 5 & Woolen cloth (D19) & Not Tested & 95.6 \\
\hline 6 & $\begin{array}{c}\text { Pressed calf leather } \\
\text { (D24) }\end{array}$ & 100 & 100 \\
\hline 7 & Beach sand (D29) & 85 & 90.8 \\
\hline 8 & Water (D38) & 100 & 100 \\
\hline 9 & Wood grain (D68) & 100 & 98.8 \\
\hline 10 & Raffia (D84) & 100 & 96.8 \\
\hline 11 & Plastic bubbles(D112) & Not Tested & 86.4 \\
\hline 12 & Gravel & Not Tested & 99.06 \\
\hline 13 & Field Stone & 76.25 & 85.25 \\
\hline 14 & Brickwall & Not Tested & 95.45 \\
\hline 15 & Pigskin (D92 H.E.) & 92.5 & 91.56 \\
\hline
\end{tabular}

Table 1: Comparison of result

\subsection{Feature Extraction}

Features are computed with the algorithm discussed in section iv of a block. This process generates 384 features ( 2 images ( 1 original +1 complement $) * 4$ combinations $((\mathrm{A}, \mathrm{H}),(\mathrm{A}, \mathrm{V})$, $(\mathrm{A}, \mathrm{D}),(\mathrm{A}, \operatorname{abs}(\mathrm{V}-\mathrm{H}-\mathrm{D}))) * 3$ features (correlation coefficient, mean and standard deviation)*16 histograms. This forms a feature vector of 90 blocks of a single category. Then a feature matrix of fifteen categories is prepared to train the neural network. Similarly a feature vector for test blocks is calculated.

\subsection{Classification Results}

The experiments were conducted using neural network and the results are shown in Table 1 which compares performance of the classification produced by binary feature selection [18]. Feature extraction method in this paper has boosted the performance in the Beach sand texture by 5.8 percent. Performance is also improved in Field stone texture. The proposed system is simple and computationally less expensive than the method in [18], which employs computationally expensive genetic algorithm. Our experiments also include some new textures Herringbone, Woolen cloth, Plastic bubbles, Gravel and Brickwall.

\section{CONCLUSION}

In the proposed image classification system we have introduced new approach using Haar wavelet decomposition and Back Propagation Neural Network. We used the correlation coefficient, mean and standard deviation features of the various combinations of coefficients produced by the wavelet transform. A number of texture images not considered in the work [18] have been analyzed in this work and have been found working within the range 86.2- 99.06\% of the performance. This work may further be extended with feature extraction using curvelet and ridgelet transform.

\section{ACKNOWLEDGEMENT}

The authors would like to thank Faculty of Engineering \& Technology of Mody Institute of Technology \& Science, Lakshmangarh for providing necessary facilities to carry out this research work.

\section{REFERENCES}

[1] Gong, P. and Howarth, P.J.,"Frequency-based contextual classification and gray-level vector reduction for landuse identification" Photogrammetric Engineering and Remote Sensing, 58, pp. 423-437. 1992.

[2] Kontoes C., Wilkinson G.G., Burrill A., Goffredo, S. and Megier J, "An experimental system for the integration of GIS data in knowledge-based image analysis for remote sensing of agriculture" International Journal of Geographical Information Systems, 7, pp. 247-262, 1993.

[3] Foody G.M., "Approaches for the production and evaluation of fuzzy land cover classification from remotely-sensed data” International Journal of Remote Sensing, 17,pp. 1317-1340 1996.

[4] San miguel-ayanz, J. and Biging, G.S," An iterative classification approach for mapping natural resources from satellite imagery" International Journal of Remote Sensing, 17, pp. 957-982, 1996. 
[5] Aplin P., Atkinson P.M. and Curran P.J," Per-field classification of land use using the forthcoming very fine spatial resolution satellite sensors: problems and potential solutions" Advances in Remote Sensing and GIS Analysis, pp. 219-239,1999.

[6] Stuckens J., Coppin P.R. and Bauer, M.E., ’Integrating contextual information with per-pixel classification for improved land cover classification" Remote Sensing of Environment, 71, pp. 282-296, 2000.

[7] Mojsilovic A., Popovic M.V. and D. M. Rackov, "On the selection of an optimal wavelet basis for texture characterization", IEEE Transactions on Image Processing, vol. 9, pp. 2043-2050, December 2000.

[8] M. Unser, "Texture classification and segmentation using wavelets frames", IEEE Transactions on Image Processing, vol. 4, pp. 1549-1560, November 1995.

[9] Bishop H., Schneider, W. Pinz A.J., "Multispectral classification of Landsat -images using Neural Network”, IEEE Transactions on Geo Science and Remote Sensing. 30(3), 482-490, 1992.

[10] Heerman.P.D.and Khazenie, "Classification of multi spectral remote sensing data using a back propagation neural network", IEEE trans. Geosci. Remote Sensing, 30(1),81-88,1992.

[11] Hepner.G.F., “Artificial Neural Network classification using minimal training set: comparision to conventional supervised classification" Photogrammetric Engineering and Remote Sensing, 56,469-473,1990.
[12] Mohanty.k.k. and Majumbar. T.J.,"An Artificial Neural Network (ANN) based software package for classification of remotely sensed data", Computers and Geosciences, 81-87, 1996

[13] Shamik Tiwari, Ajay Kumar Singh and V P Shukla, "Statistical Moments based Noise Classification using Feed Forward Back Propagation Neural Network", International Journal of Computer Applications 18(2):36-40, March 2011.

[14] Stéphane G. Mallat, 1999, “A wavelet tour of signal processing”, Academic Press, 1999.

[15] Chin-Chen Chang, Jun-Chou Chuang and Yih-Shin Hu, "Similar Image Retrieval Based On Wavelet Transformation, International Journal Of Wavelets", Multiresolution And Information Processing, Vol. 2, No. 2, 2004, pp.111-120, 2004.

[16] Charles K. Chui, "An Introduction to Wavelets", Academic Press, 1992.

[17] Kumar S., "Neural Networks: A Classroom Approach", 1st Edn., Tata Mc-Graw Hill Publications, ISBN: 0-07048292-6, pp: 169,2004.

[18] Montiel E., Aguado A. S., M. S. Nixon, "Texture classification via conditional histograms", Pattern Recognition Letters, 26, pp. 1740-1751(2005).

[19] Brodatz P., "Textures: A Photographic Album for Artist \& Designers", New York: Dover, New York, 1966. 